\title{
DNA Hash Pooling and its Applications
}

\author{
Dennis Shasha \\ Courant Institute of Mathematical Sciences \\ New York University \\ shasha@cs.nyu.edu \\ Martyn Amos \\ Department of Computing and Mathematics \\ Manchester Metropolitan University \\ M.Amos@mmu.ac.uk
}

May 24, 2007

\begin{abstract}
In this paper we describe a new technique for the characterisation of populations of DNA strands. Such tools are vital to the study of ecological systems, at both the micro (e.g., individual humans) and macro (e.g., lakes) scales. Existing methods make extensive use of DNA sequencing and cloning, which can prove costly and time consuming. The overall objective is to address questions such as: (i) (Genome detection) Is a known genome sequence present at least in part in an environmental sample? (ii) (Sequence query) Is a specific fragment sequence present in a sample? (iii) (Similarity Discovery) How similar in terms of sequence content are two unsequenced samples?

We propose a method involving multiple filtering criteria that result in "pools" of DNA of high or very high purity. Because our method is similar in spirit to hashing in computer science, we call the method DNA hash pooling. To illustrate this method, we describe examples using pairs of restriction enzymes. The in silico empirical results we present reflect a sensitivity to experimental error. The method requires minimal DNA sequencing and, when sequencing is required, little or no cloning.
\end{abstract}

\section{Introduction}

Biologists often examine large and diverse populations of organisms (for example, molecules, microbes or plants). This is particularly the case in fields such as microbial ecology, which studies the interactions between living microorganisms (such as algae, or bacteria) and their environment. One of the most significant and challenging problems in these areas of biology is to quantify the overall diversity of a given population. This task is often made even more difficult by the fact that many "wild" organisms resist laboratory cultivation (and, thus, have unknown phenotypes and their genomes are unknown), or may be present in a population in relatively low numbers.

The study of metagenomics has emerged in recent years $8,10,16,18$ to perform what has been described as "environmental forensics," including the 
quantification of relative abundances of known species, and the estimation of the number of "unknown" species in a given environment 10. The potential impact of this new field is huge, with applications ranging from medicine to agriculture and biotechnology. Further the insights gained will be of significant assistance in furthering our understanding of biodiversity in both new and familiar environments, such as frozen Antarctic lakes and the human gut [8].

Metagenomic analysis currently involves the extraction of DNA from an environmental sample, cloning of the DNA into a suitable "vector", insertion of the vector into a host bacterium and then screening the resulting transformed bacteria [8. Screening may occur on the basis of gene expression using microarrays [18] or some other trait, such as antibiotic production [15, or the bacteria may simply be sequenced at random. We now briefly describe the first (microarray) and third (sequence-based) methods.

\section{$1.1 \quad$ Microarrays}

Microarrays 14 permit the study of gene expression and the detection of mutations. "Similar to the situation in which microprocessors have increased the speed of computation, microarray-based genomic technologies have revolutionized genetic analysis of biological systems" [18. However, they tend to be used for relatively pure, or homogenous samples, and their applicability at the community level is less well-understood. In order to identify unknown bacterial strains, the microarray requires a "probe" taken from a related strain, which may or may not be available. In addition, the cost of microarray equipment is, in the short to medium term, high for non-trivial studies.

\subsection{Sequencing}

Sequence-based approaches, on the other hand are guided by existing genomic knowledge-bases, and we briefly describe two of these here.

\subsubsection{Environmental Gene Tags}

The assembly of genomes from complex communities currently "demands enormous sequencing expenditure for the assembly of even the most predominant members" [16]. Because of this difficulty, borne out by initial studies by Tringe et al. [16, the authors decided to employ an alternative, "gene-centric" approach that does not attempt to attribute genes obtained to any particular genome. They obtained their initial dataset by taking four sets of samples, one from agricultural soil, and three from whale carcasses. Samples were then partitioned into bacteria, archea or eukaryotes using PCR-amplified rRNA libraries. Genomic small-insert libraries were then sequenced from each sample (100 million base pairs from the soil and 25 million base pairs from each whale sample). These sequences, derived from different population members, were termed "Environmental Gene Tags" (EGTs), since they may encode regions of functional genes that are necessary for survival in a particular environment. Different environment types will exhibit unique EGT "fingerprints", containing genes derived from many different genomes. The study showed that two whale carcasses, located $8000 \mathrm{~km}$ apart, nontheless had very similar EGT patterns. Thus, one may determine the type of environment from this fingerprinting technique. 


\subsubsection{MEGAN}

In a recent study, Huson et al present an approach 10 to the problem of genomic assembly in which the authors compare sequenced data to existing databases. Specifically, the set of DNA sequences obtained by random shotgun sequencing from the environmental sample is run against known sequences using BLAST. The resulting meta-data is then provided as input to the MEGAN package, which estimates and explores the taxonomical content of the data set. This may be a good technique to obtain the most abundant species in a sample, but will have difficulty locating rare sequences of interest. One of the themes of our approach is to hunt systematically for signs of a genome of interest.

\subsection{Implications}

The existing approaches require significant sequencing effort. In 2007, DNA sequencing costs approximately 1 (U.S.) cent per base [5. Even for a relatively simple community study on the drainage region of an acid mine, roughly 15 million bases were sequenced in order to obtain the required metagenomic data [16], at a cost (today) of approximately $\$ 150,000$. A soil study, requiring at least 50 million bases, might then cost half a million US dollars.

Fortunately, sequencing is not always necessary as a first step. Molecular techniques that work at the whole sequence level may be used to reduce the initial complexity of a sample population. One tool commonly employed is "GC fractionation" 9], which works along the principles of a molecular "sieve", sorting strands according to their relative GC content (guanine and cytosine being heavier than their counterparts adenine and thymine). This may be effective when trying to partition a sample into eukaryotic and bacterial sets, since eukaryotic DNA tends to have a much lower GC content (e.g, we selected two complete bacterial genome sequences, A (Escherichia coli K12) and B (Shigella boydii Sb227) for early studies; each of these had a GC content of roughly $51 \%$, while the human genome is made up of around $45 \% \mathrm{GC}$ and that of the mouse roughly 44\%). However, such a relatively crude tool rapidly proves ineffective when dealing with shorter sequences, where we may only possess genomic fragments within our sample. For bacterial sequence A, when taking 200 random consecutive sequences of length 50,000, we obtained a GC content ranging from $46.7 \%$ to $53.3 \%$ with the $90 \%$ confidence interval ranging from $47.3 \%$ to $52.8 \%$.

Preliminary work on estimating the complexity of a heterogenous population of DNA strands (without using sequencing) is reported in [7. This paper, motivated in part by the authors' earlier work on DNA-based computing 1, reports initial experimental investigations into the use of basic laboratory methods (combined with probability theory) to estimate the complexity of a tube of strands. Faulhammer et al. digested their initial tube with a set of restriction enzymes with recognition sites differing in sequence and of length $4 \leq k \leq 8$. The contents of the tube were then visualised in a gel, and the number of distinct bands observed used to obtain an estimate of the number of different strands.

Our proposal uses some of the same basic laboratory methods, but it differs from that of [7] in several important ways:

1. Rather than simply counting the number of different restriction fragments obtained, we use the lengths themselves to obtain a partition of segments. 
2. We use the resulting data structure both in silico and in vitro to compare different DNA "tubes".

3. We use multiple levels of restriction enzyme digestion.

This basic approach (the use of restriction enzymes to digest a population sample, followed by analysis of the fragment size) also underpins an early variant of the well-known technique of DNA fingerprinting [11. Restriction fragment length polymorphisms (RFLPs) [6] provide a technique by which organisms may be differentiated by comparing the patterns obtained by digesting a certain portion of their DNA. If two organisms differ in the distance between restriction sites, the length of the fragments produced will differ (i.e., be polymorphic) when the DNA is digested. However, this method is generally only useful when the population sample is relatively homogeneous (e.g., one wishes to distinguish between members of the same species).

In the rest of the paper, we present and evaluate a simple and powerful technique called DNA hash pooling. We conclude with a discussion of plans for future theoretical and experimental work.

\section{DNA Hash Pooling}

In computer science, hashing 12 maps a relatively small set from a large domain (e.g., 10,000 integers ranging in value from 0 to one billion) to a small domain (e.g,. the set of integers from 1 to 5000) through a mathematical hash function. Applications of hashing include cryptography, error correction, authentication and identification. A typical hash function is modulus (i.e., remainder). For example, $7 \bmod 5=2$ because 2 is the remainder after dividing 7 by 5 . For the same reason, $28 \bmod 5=3$. A hash data structure based on "mod 5 " will map 28 to bucket (or pool) 3, 7 to pool 2, 12 to pool 2, 59 to pool 4, and so on. There are many variants of hashing, some of which entail hashing each pool resulting from the first hash function in order to get "purer" pools, and then using the combined hash results to generate an item "label". For example, using a second hash function, based on "mod 7", 28 would map to 0 and 53 to 5 . Thus the full "label" of 28 would be $(3,0)$ because $28 \bmod 5=3$ and $28 \bmod 7=0$. By contrast, the label of 53 would be $(3,4)$ because $53 \bmod 5=3$ and $53 \bmod 7$ is 4. Associated with each unique label is a pool having a relatively small number of distinct values.

DNA hash pooling or hash pooling for short is the analogous operation on DNA. The "hash functions" in this scenario correspond to biological operations that give rise to distinctive and quantifiable "fingerprints" (e.g., measurement of GC content followed by digestion by a set of restriction enzymes). The label components correspond to the "values" obtained by application of the hash functions (e.g. GC content and fragment length).

In silico, our method involves simulating these operations on known sequences (typically though not necessarily of entire genomes) and characterizing different portions of those sequences from the result(s). In vitro, our method involves performing the bench-based operations and sequencing only those pools that are likely to be pure (this is, unique to one genome), or otherwise of interest.

For concreteness, this paper focusses on hash pooling based solely on restriction enzymes. The basic operations are 


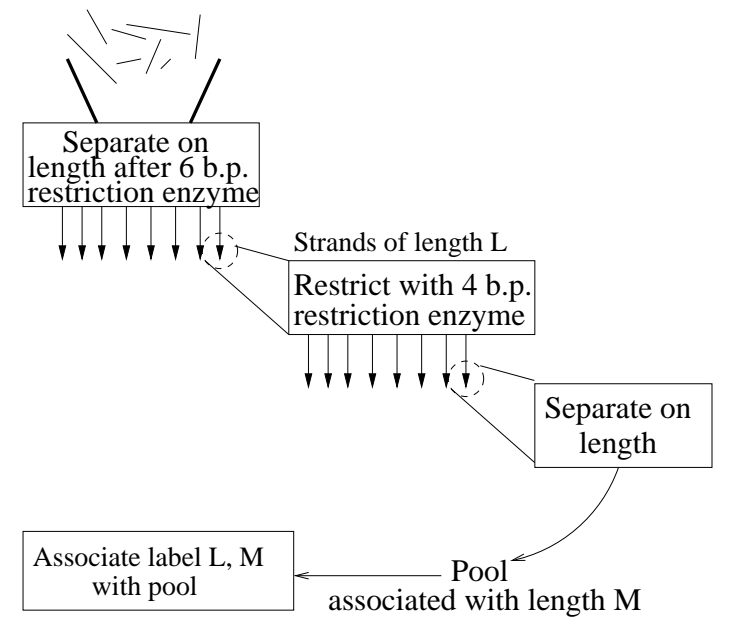

Figure 1: Two-stage hash pooling

1. Apply a six base-pair (base pair) restriction enzyme to a sequence, yielding a set of fragments.

2. Partition those fragments based on length (perhaps approximately), using a technique such as gel electrophoresis.

3. Apply a four base pair restriction enzyme to a selected subset of partitions and separate on length again.

4. Sequence selected lengths.

Each resulting pool is therefore associated with a label consisting of two lengths, the first based on a six base pair restriction enzyme and the second based on a four base pair restriction enzyme (Figure 1). 
The procedure may be described for $\mathrm{K}$ stages in pseudo-code as follows:

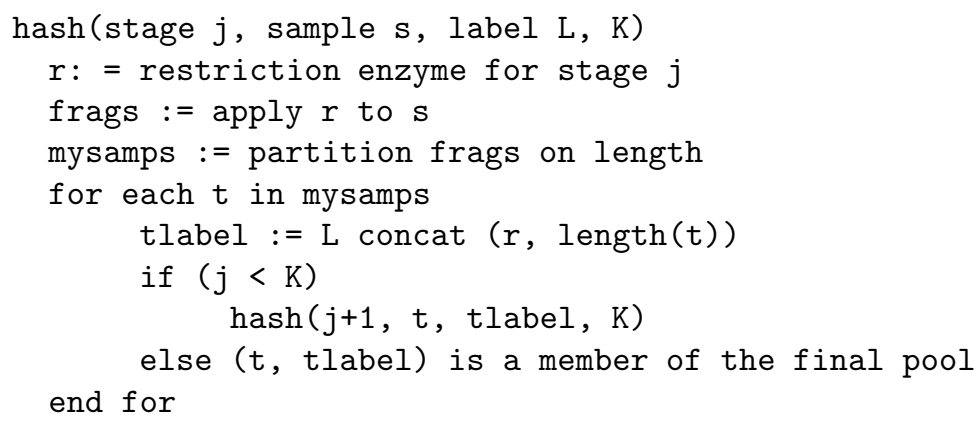

Pseudo-code for $\mathrm{K}$ stage hash pooling based on restriction enzymes. The initial call on an initial sequence orig would be hash(1, orig, null, K), where null is the empty label.

For example, consider the genomic sequence of bacterium A (E. coli K12). If we cut A using the enzyme SmaI (recognising CCCGGG), take the pool corresponding to length 264, cut that pool with RsaI (GTAC) and take the pool of length 31 , we get a pool having label $(264,31)$. It happens to have a single member with the sequence CTATCCGCTCAATGAGTCGGTCGCCATTGCC. By contrast, the pool with label $(770,207)$ has three different sequences. For some applications, we will want pools having singletons (i.e., a set with only a single element) in order to obtain a pure sequence without the need for cloning.

One may object that separating fragments by length entails a certain inaccuracy imposed by the laboratory technique; a reasonable estimate of this error may be plus or minus 10 base pairs 3 . In this case, in order to obtain a pure sample, we may be interested in finding a pool whose label has no "10 base pair-neighbors." The labels $L$ and $L^{\prime}$ are 10 base pair-neighbors if (i) the first component of $L$ and the first component of $L^{\prime}$ are different but differ by 10 or less $\left(0<\left|L[0]-L^{\prime}[0]\right| \leq 10\right)$; or (ii) the first component of $L$ and $L^{\prime}$ are the same but the second components differ by 10 or less $\left(0<\left|L[1]-L^{\prime}[1]\right| \leq 10\right)$. For $E$. coli K12, the labels $(188,59)$ and $(188,106)$, for example, have no 10 base pair-neighbors.

\section{$3 \quad$ Experiments}

Having presented our formal framework, we can now present several applications and our in silico empirical results.

\subsection{Genome Detection}

The first question we ask is the following: given a tube, $T$, of unknown DNA (perhaps from an environmental sample) and a genome whose sequence is known, are "reasonably sized" portions of that genome present in $T$, even if in small concentrations? (Figure 2)

A "reasonably sized" portion is a sequence of length at least 200,000 base pairs (or roughly $5 \%$ of the length of a bacterial genome.) This might be used for the detection of bacterial pathogens in food, for example. In what follows, we 


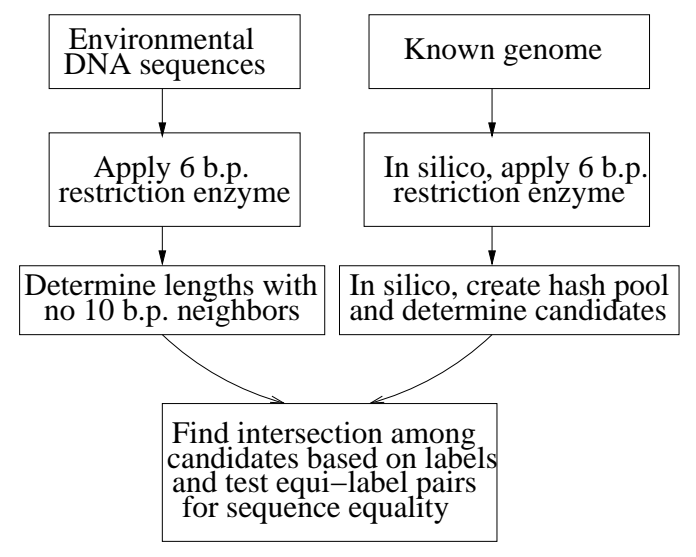

Figure 2: Genome sequence detection

used bacterium A, E. coli $\mathrm{K} 12$, which is often used as an indicator organism in the detection of faecal contamination. Our in silico result involved the following steps:

1. Compute the candidate set of A, consisting of possibly non-singleton pools having no 10 base pair neighbors. There were 3,567 candidates. This gives us a "comparison library" of pools. It is important to note that this step is purely computational and can be computed just once for any combination of known genome and restriction enzyme set.

2. We simulate the unknown sample $T$ by taking a 200,000 consecutive base pair subsequence of A (with the start position taken uniformly at random) and combining it with a sequence of length four times that of A (generated pseudo-randomly to have the same GC content as A).

3. We then compute the resulting candidate set of hash pools, based on no 10 base pair neighbors.

Having tried this 20 times, we found, on average, 2,000 pools in the second candidate set. On average the two sets had an intersection of cardinality 71 based on their labels. When labels were equal, $99.8 \%$ of the time there was a match of the sequence and the sequence came from that 200,000 consecutive base pair subsequence, giving a precision of $99.8 \%$. This implies that one may be able to avoid sequencing if one finds enough common candidates. Further the recall was $100 \%$ in that we always found a matching label.

When applying this in a laboratory setting, there is the significant question of whether this operation many separate DNA extractions and applications of a restriction enzyme. Fortunately, the answer is no. For each of the 20 tests, first the six base pair restriction enzyme was used. This gave a collection of fragment lengths. On average only 5.8 of those lengths had no 10 base pair neighbors and had lengths similar to the lengths of the candidates from A. 1 So on average only 5.8 fragment lengths required extraction. Of those, 4.7 (on average) yielded matching sequences. So, if this were done in vitro, approximately

\footnotetext{
${ }^{1}$ Typical lengths were between 7,000 base pairs and 39,000 base pairs
} 
70 common strands would be found using one application of SmaI and under six applications of RsaI. Virtually all (99.8tested strands would be shown to be equal, so sequencing would not even be necessary.

This experiment shows that in silico hash pooling on a known genome can identify pools to look for in a sample, such that those pools have a strong likelihood of containing a subsequence of the known genome. Thus, we can see this method as an improvement over random sampling, and can be used even if the bacterium of interest is relatively rare in the sample.

\subsection{Sequence Query}

Here we address the question: given a query sequence, is that sequence present, at least in part, in the tube? This might be used to look for the presence of a pathogen, for example. This question is clearly related to the previous one. In fact, an experiment similar to that used to address the first question serves as an illustration: suppose the sample under scrutiny contains A plus a lot of other assorted DNA (e.g. the full genome of A amongst a pseudo-random sequence four times the length of the A sequence and having the same GC content). Then, 20 times, we take a random query subsequence of length 200,000 from A and see if we can find matching parts in the sample tube.

The sample tube (A sequence plus a random sequence four times $\mathrm{A}$ in length with no 10 base pair neighbors) has 3,516 candidate pools. The average 200,000 base pair subsequence of $\mathrm{A}$ has about 200 candidates. In our 20 experiments, whenever two labels are equal, the corresponding sequences matched $100 \%$ of the time (precision of sequence matching given label match of 100\%). This is not guaranteed to hold always of course, but again shows that even without sequencing one can be quite sure that sequences will match if labels match. Recall was not as good, as we found matching labels in $17 / 20$ or 85

As in the first experiment, the six base pair restriction enzyme would cut the fragments into certain lengths, but, on the average, only 2.3 of those lengths (ranging from 10,000 base pairs to 30,000 base pairs) would have the properties that (i) they had no 10 base pair neighbors and (ii) they matched the candidates from the 200,000 base pair query sequence. Thus, on the average, under three extractions need to be taken and then digested by the four base pair restriction enzyme.

We also tried the negative case when the query sequence was nowhere present in the sample. In that case, on the average, after cutting with the six base pair restriction enzyme, on the average, under one of those lengths had the properties that (i) they had no 10 base pair neighbors and (ii) they matched the candidates from the 200,000 base pair query sequence. When extracted and digested by the four base pair restriction enzyme, there were no matching labels (other than a single label whose final fragment length was only 4 ). So this technique does not throw up false positives.

\subsection{Similarity Discovery}

Here we consider the problem: given two tubes of DNA, do they contain strands that are the same or very similar? This might be useful when comparing samples of unsequenced genomes. In this case, we cannot compute candidate pools that have no 10 base pair neighbors using known genomes. Instead, we have to 


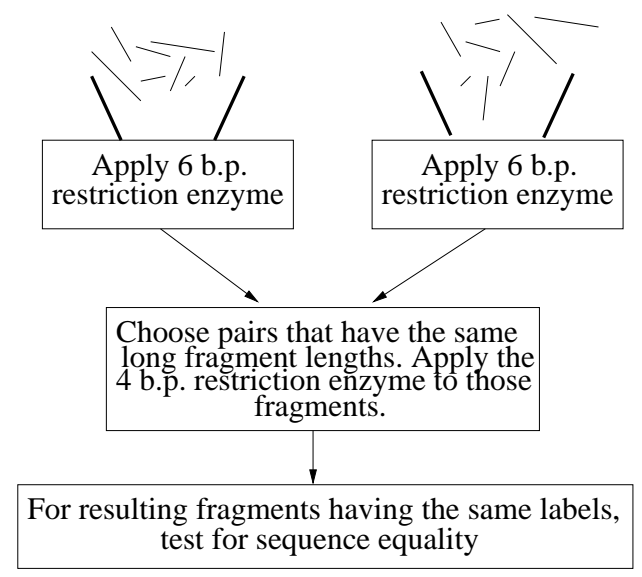

Figure 3: Sample comparison

measure them. Sometimes we may not know whether the sample contains known sequences. If it does, we can use the techniques in the Subsequence Detection subsection above to find out which known genomes each sample contains and then see which are the same.

Let us assume however that the sample contains no known genomes (or that we want to detect commonalities besides those among known genomes). Our strategy will be to choose the most likely pairs to study by focussing on "unusual" labels (Figure [3). We therefore performed the following in silico experiment:

1. Take a 200,000 base pair sequence, target, with the same GC content as $\mathrm{A}$, plus a random sequence four times the size of $\mathrm{A}(4 \times 4.7 M b=\approx 20 M b)$, with the same average GC content as A.

2. For the second sample, we use the same 200,000 base pair sequence target plus another random sequence four times the size of $\mathrm{A}$, with the same average GC content. Thus the target in each sample is 200,000 base pairs long, just $1 \%$ of the roughly roughly 20 million for the entire sequence present in each tube.

Now the question is this: in which pools should we look for common strands? That is, is it better to look at pools where the six base pair restriction enzyme has cut strands of length approximately 4,000 (the expected value) or much longer? The in silico answer is obvious in retrospect: to find common strands, the best pools to look at are ones corresponding to long lengths when cut by the first restriction enzyme. Thus the procedure is this:

1. Cut each sample with the six base pair restriction enzyme, then find all lengths that are the same (within an accuracy of 10 base pairs).

2. On the upper quartile of those lengths (approximately 235 of them), apply the four base pair restriction enzyme.

Of those fragments that have the same lengths (within 10 base pairs) for both the first and second restriction enzymes, between $4 \%$ and $7 \%$ are the same 
sequence over the 20 experiments that we tried. Other quartiles are about a factor of 10 less good. On the other hand, if we look at dectiles $(1 / 10)$, then the upper $1 / 10$ of the lengths (from 10,210 to 24,550) gives a hit ratio of about $14 \%$, and only 94 lengths from the six base pair restriction enzyme require an application of the second restriction enzyme.

If we have already identified known genomes that the two tubes share in common, then we should avoid labels that correspond to those.

\section{Implementation Issues}

In this section we give a brief overview of the two main laboratory tools from which our basic operations are built: restriction enzymes (for chopping DNA into sections) and gel electrophoresis (for sorting fragments according to length). The descriptions are taken from 2 .

\subsection{Restriction Enzymes}

Restriction endonucleases 17 (often referred to as restriction enzymes) recognize a specific sequence of DNA known as a restriction site. Any DNA that contains the restriction site within its sequence is cut by the enzyme at that point.

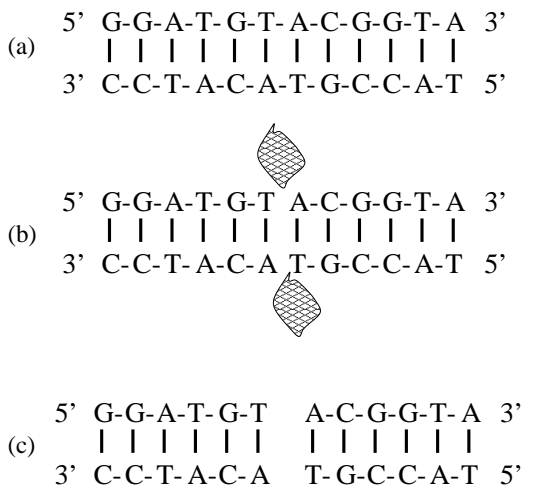

Figure 4: (a)Double-stranded DNA. (b) DNA being cut by RsaAI. (c) The resulting blunt ends

For example, the double-stranded DNA in Fig. 4 $4 \mathrm{a}$ is cut by restriction enzyme $R s a \mathrm{I}$, which recognizes the restriction site $G T A C$. The enzyme breaks (or "cleaves") the DNA in the middle of the restriction site (Fig. 4b). The exact nature of the break produced by a restriction enzyme is of great importance. Some enzymes like RsaI (mentioned earlier) leave "blunt" ended DNA (Fig. 45).

\subsection{Gel Electrophoresis}

Gel electrophoresis is an important technique for sorting DNA strands by size [4. Electrophoresis is the movement of charged molecules in an electric field. Since DNA molecules carry a negative charge, when placed in an electric field they tend to migrate toward the positive pole. The rate of migration of a molecule 


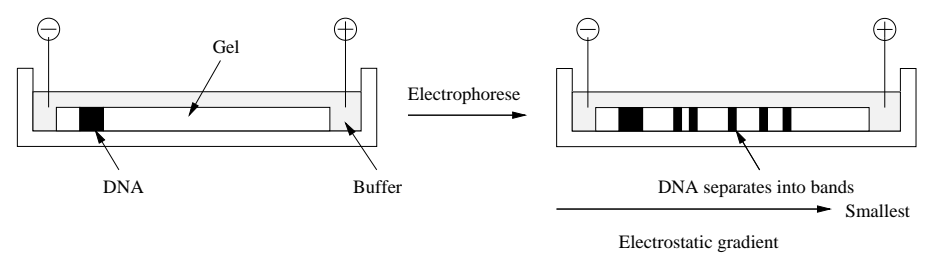

Figure 5: Gel electrophoresis process

in an aqueous solution depends on its shape and electric charge. Since DNA molecules have the same charge per unit length, they all migrate at the same speed in an aqueous solution. However, if electrophoresis is carried out in a gel (usually made of agarose, polyacrylamide, or a combination of the two), the migration rate of a molecule is also affected by its size 2 This is due to the fact that the gel is a dense network of pores through which the molecules must travel. Smaller molecules therefore migrate faster through the gel, thus sorting them according to size.

A simplified representation of gel electrophoresis is depicted in Fig. 5. The DNA is placed in a well cut out of the gel, and a charge applied.

Once the gel has been run (usually overnight), it is necessary to visualize the results. This is achieved by staining the DNA with the fluorescent dye ethidium bromide and then viewing the gel under ultraviolet light. At this stage the gel is usually photographed.

One such photograph is depicted in Fig. 6. Gels are interpreted as follows; each lane (1-7 in our example) corresponds to one particular sample of DNA (we use the term tube in our abstract model). We can therefore run several tubes on the same gel for the purposes of comparison. Lane 7 is known as the marker lane; this contains various DNA fragments of known length, for the purpose of calibration. DNA fragments of the same length cluster to form visible horizontal bands, the longest fragments forming bands at the top of the picture, and the shortest ones at the bottom. The brightness of a particular band depends on the amount of DNA of the corresponding length present in the sample. Larger concentrations of DNA absorb more dye, and therefore appear brighter. One advantage of this technique is its sensitivity - as little as $0.05 \mu \mathrm{g}$ of DNA in one band can be detected as visible fluorescence.

The size of fragments at various bands is shown to the right of the marker lane, and is measured in base pairs. In the photograph, the largest band resolvable by the gel is 2,036 base pairs long, and the shortest one is 134 base pairs long. Moving right to left (tracks $6-1$ ) is a series of PCR reactions which were set up with progressively diluted target DNA (134 base pairs) to establish the sensitivity of a reaction. The dilution of each tube is evident from the fading of the bands, which eventually disappears in lane 1.

\footnotetext{
[13.

${ }^{2}$ Migration rate of a strand is inversely proportional to the logarithm of its molecular weight
} 


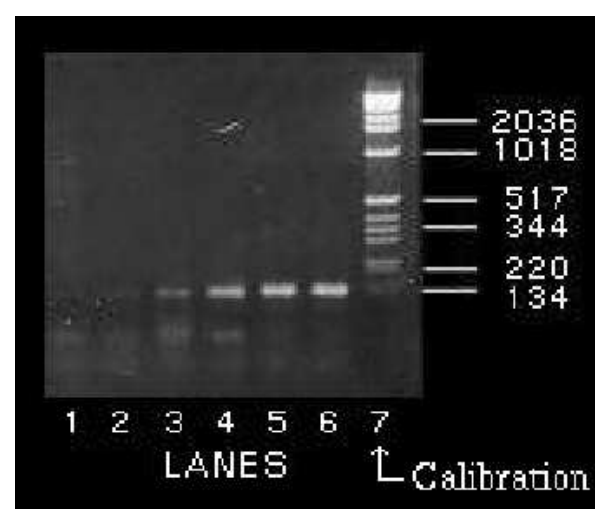

Figure 6: Gelelectrophoresis photograph

\section{Conclusions}

DNA Hash pooling is a method to simplify many problems in metagenomics. It gives the experimenter the ability to query for known sequences and genomes in a sample or to find common sequences from unknown genomes in two or more samples even if the identified sequences are rare. The version of the technique described in this paper involves a small number of steps of the form: extract DNA of a certain length, apply a restriction enzyme to it, and measure the lengths of the results. In most cases, sequencing is unnecessary and, where it is, cloning is not. The main technical challenge is to get reasonably accurate measurements of length.

The main future work we anticipate is to validate the technique and then extend the method as new application scenarios present themselves.

\section{Acknowledgments}

Shasha's work has been partly supported by the U.S. National Science Foundation under grants IIS-0414763, DBI-0445666, N2010 IOB-0519985, N2010 DBI0519984, DBI-0421604, and MCB-0209754. This support is greatly appreciated.

\section{References}

[1] Leonard M. Adleman. Molecular computation of solutions to combinatorial problems. Science, 266:1021-1024, 1994.

[2] Martyn Amos. Theoretical and Experimental DNA Computation. Springer, 2005 .

[3] Annelise E. Barron, Harvey W. Blanch, and David S. Soane. A transient entanglement coupling mechanism for dna separation by capillary electrophoresis in ultradilute polymer solutions. Electrophoresis, 15:597$615,1994$.

[4] T.A. Brown. Genetics: A Molecular Approach. Chapman and Hall, 1993. 
[5] Francis S. Collins and Anna D. Barker. Mapping the cancer genome. Scientific American, February 2007.

[6] T.E. Dowling, C. Moritz, and J.D. Palmer. Nucleic acids II: restriction site analysis. In D.M. Hillis and C. Moritz, editors, Molecular systematics, pages 250-317. Sinauer Associates, 1990.

[7] Dirk Faulhammer, Richard J. Lipton, and Laura F. Landweber. Counting DNA: estimating the complexity of a test tube of DNA. BioSystems, 52(13):193-196, 1999.

[8] Jo Handelsman. Metagenomics: Application of genomics to uncultured microorganisms. Microbiology and Molecular Biology Reviews, 68(4):669 $685,2004$.

[9] W.E. Holben and D. Harris. DNA-based monitoring of total bacterial community structure in environmental samples. Molecular Ecology, 4(5):27-31, 1995.

[10] Daniel H. Huson, Alexander F. Auch, Ji Qi, and Stephan C. Schuster. MEGAN analysis of metagenomic data. Genome Research, 17:377-386, 2007.

[11] A.J. Jeffreys, V. Wilson, and S.L. Thein. Hypervariable 'minisatellite' regions in human DNA. Nature, 314:67-73, 1985.

[12] Donald Knuth. The Art of Computer Programming, Volume 3: Sorting and Searching. Addison-Wesley, second edition, 1998.

[13] R. Old and S. Primrose. Principles of Gene Manipulation, an Introduction to Genetic Engineering. Blackwell, fifth edition, 1994.

[14] M. Schena, D. Shalon, R.W. Davis, and P.O. Brown. Quantitative monitoring of gene expression patterns with a complementary DNA microarray. Science, 270(5235):467-470, 1995.

[15] P.D. Schloss and J. Handelsman. Biotechnological prospects from metagenomics. Current Opinion in Biotechnology, 14:303-310, 2003.

[16] Susannah Green Tringe et al. Comparative metagenomics of microbial communities. Science, 308:554-557, 2005.

[17] J. Williams, A. Ceccarelli, and N. Spurr. Genetic Engineering. Bios Scientific Publishers, 1993.

[18] Jizhong Zhou. Microarrays for bacterial detection and microbial community analysis. Current Opinion in Microbiology, 6:288-294, 2003. 


\title{
DNA Hash Pooling and its Applications
}

\author{
Dennis Shasha and Martyn Amos
}

April 30

\begin{abstract}
In this paper we describe a new technique for the characterisation of populations of DNA strands. Such tools are vital to the study of ecological systems, at both the micro (e.g., individual humans) and macro (e.g., lakes) scales. Existing methods make extensive use of DNA sequencing and cloning, which can prove costly and time consuming. The overall objective is to address questions such as: (i) Is a known genome sequence present in an environmental sample? (ii) Is a specific fragment sequence present in a sample? (iii) How similar in terms of sequence content are two samples? (iii) Does a sample contain a desired concentration of a long sequence?

We aim to answer these questions using minimal DNA sequencing and, when sequencing is required, using little or no cloning. Furthermore, we seek to design a method that is economical and practical. We propose a probabilistic method involving multiple filtering criteria that result in "pools" of DNA of high or very high purity. Because our method is similar in spirit to hashing in computer science, we call the method hash pooling. To illustrate this method, we describe examples using pairs of restriction enzymes. Our empirical results are in silico but reflect a sensitivity to experimental error.
\end{abstract}

\section{Introduction}

Biologists often examine large and diverse populations of organisms (for example, molecules, microbes or plants). This is particularly the case in fields such as microbial ecology, which studies the interactions between living microorganisms (such as algae, or bacteria) and their environment. One of the most significant and challenging problems in these ares of biology is to quantify the overall $d i$ versity of a given population. This task is often made even more difficult by the fact that many "wild" organisms resist laboratory cultivation (and, thus, have unknown phenotypes and their genomes are unknown), or may be present in a population in relatively low numbers. In this paper, we present a novel approach to solving this problem.

The rest of the paper is organized as follows. We first motivate what follows by introducing the new field of metagenomics, which is concerned mainly with population-level genetic studies. By describing existing methods for characterizing DNA samples, we highlight potential shortcomings in their methodology or applicability. We then present an alternative technique, which we call $D N A$ hash pooling, describe the method and the results of preliminary in silico studies, 
and conclude with a discussion of plans for future theoretical and experimental work.

\section{Metagenomics}

The study of metagenomics has emerged in recent years [?, ?, ?, ?] to perform what has been described as "environmental forensics", including the quantification of relative abundances of known species, and the estimation of the number of "unknown" species in a given environment [?]. The potential impact of this new field is huge, with applications ranging across medicine, agriculture and biotechnology, and the insights gained will be of significant assistance in furthering our understanding of biodiversity in both new and familiar environments, such as frozen Antarctic lakes and the human gut [?].

Metagenomic analysis involves the extraction of DNA from an environmental sample, cloning of the DNA into a suitable "vector", insertion of the vector into a host bacterium and then screening the resulting transformed bacteria [?]. Screening may occur on the basis of gene expression using microarrays [?] or some other trait, such as antibiotic production [?], or the bacteria may simply be sequenced at random. We now briefly describe the first (microarray) and third (sequence-based) methods.

\section{$2.1 \quad$ Microarrays}

Microarrays [?] permit the study of gene expression and the detection of mutations. "Similar to the situation in which microprocessors have increased the speed of computation, microarray-based genomic technologies have revolutionized genetic analysis of biological systems" [?]. However, they tend to be used for relatively pure, or homogenous samples, and their applicability at the community level is less well-understood. In order to identify unknown bacterial strains, the microarray will still require a "probe" taken from a related strain, which may or may not be available. In addition, the cost of microarray equipment may prove prohibitive for non-trivial studies in the short to mid-term.

\subsection{Sequencing}

Sequence-based approaches, on the other hand are guided by existing genomic knowledge-bases, and we briefly describe two of these here.

\subsubsection{Environmental Gene Tags}

The assembly of genomes from complex communities may well "demand enormous sequencing expenditure for the assembly of even the most predominant members" [?], and may well prove to be infeasible for most communities in the forseeable future. Because of this difficulty, borne out by initial studies by Tringe et al. [?], the authors decided to employ an alternative, "gene-centric" approach, without attributing genes obtained to any particular genome. They obtained their initial dataset by taking four sets of samples, one from agricultural soil, and three from whale carcasses. Samples were then partitioned into bacteria, archea or eukaryotes using PCR-amplified rRNA libraries. Genomic small-insert libraries were then sequenced from each sample (100 million base 
pairs from the soil and 25 million base pairs from each whale sample). These sequences, derived from different population members, were termed "Environmental Gene Tags" (EGTs), since they may encode regions of functional genes that are necessary for survival in a particular environment. Different environment types will exhibit unique EGT "fingerprints", containing genes present in many different genomes. The study showed that two whale carcasses, located $8000 \mathrm{~km}$ apart, nontheless had very similar EGT patterns.

\subsubsection{MEGAN}

In a recent study, Huson et al present an alternative approach [?] to the problem of genomic assembly in which the authors compare sequenced data to existing databases, using BLAST. The set of DNA sequences obtained from the environmental sample is run against known sequences in silico using BLAST. The set of reads, obtained by running random shotgun sequencing on the environmental sample, is run against known sequences in silico using BLAST. The resulting meta-data is then provided as input to the MEGAN package, which estimates and explores the taxonomical content of the data set. This may be a good technique to obtain the most abundant species in a sample.

\subsection{Implications}

Both of the existing approaches require significant sequencing effort. In 2007, DNA sequencing costs approximately 1 (U.S.) cent per base [?]. Even for a relatively simply community study on the drainage region of an acid mine, roughly 15 million bases were sequenced in order to obtain the required metagenomic data [?], at a cost (today) of approximately $\$ 150,000$. A soil study, requiring at least 50 million bases, might then cost half a million US dollars.

However, it may not always be necessary to perform sequencing as a first step. Molecular techniques that work at the whole sequence level may be used to reduce the initial complexity of a sample population. One tool commonly employed is "GC fractionation" [?], which works along the principles of a molecular "sieve", sorting strands according to their relative GC content (guanine and cytosine being heavier than their counterparts adenine and thymine). This may be effective when trying to partition a sample into eukaryotic and bacterial sets, since eukaryotic DNA tends to have a much lower GC content (e.g, we selected two complete bacterial genome sequences, A (Escherichia coli K12) and B (Shigella boydii Sb227) for early studies; each of these had a GC content of roughly $50 \%$, while the human genome is made up of around $45 \%$ GC and that of the mouse roughly $44 \%$ ). However, such a relatively crude tool rapidly proves ineffective when dealing with shorter sequences, where we may only possess genomic fragments within our sample. For sequence A, when taking 200 random consecutive sequences of length 50,000, we obtained a GC content ranging from $46.7 \%$ to $53.3 \%$ with the $90 \%$ confidence interval ranging from $47.3 \%$ to $52.8 \%$. If we reduce the sequence length even further, to 10,000 , then the GC content $90 \%$ confidence interval ranges from $45.8 \%$ to $54.1 \%$.

Preliminary work on estimating the complexity of a heterogenous population of DNA strands (without using sequencing) is reported in [?]. This paper, motivated in part by the authors' earlier work on DNA-based computing [?], reports initial experimental investigations into the use of basic laboratory meth- 
ods (combined with probability theory) to estimate the complexity of a tube of strands. Faulhammer et al. digested their initial tube with a set of restriction enzymes with recognition sites differing in sequence and of length $4 \leq k \leq 8$. The contents of the tube were then visualised in a gel, and the number of distinct bands observed used to obtain an estimate of the number of different strands.

Although our proposal uses the same basic laboratory methods, it differs from that of [?] in several important ways:

1. Rather than simply counting the number of different restriction fragments obtained, we use the lengths themselves to obtain a partition of segments.

2. We use the resulting data structure both in silico and in vitro to compare different DNA "tubes".

3. We may often use several levels of restriction enzyme digestion.

This basic approach (the use of restriction enzymes to digest a population sample, followed by analysis of the fragment size) underpins an early variant of the well-known technique of DNA fingerprinting [?]. Restriction fragment length polymorphisms (RFLPs) [?] provide a technique in which organisms may be differentiated by comparing the patterns obtained by digesting their DNA. If two organisms differ in the distance between restriction sites, the length of the fragments produced will differ (i.e., be polymorphic) when the DNA is digested. However, this method is generally only useful when the population sample is relatively homogeneous (e.g., one wishes to distinguish between members of the same species). We now describe our alternative scheme in more detail.

\section{DNA Hash Pooling}

In computer science, hashing [?] maps a relatively small set from a large domain (e.g., the set of integers from 0 to one billion) to a small domain (e.g,. the set of integers from 1 to 5000) through a mathematical hash function. Applications of hashing include cryptography, error correction, authentication and identification. A typical hash function is modulus (i.e., remainder). For example, 7 mod $5=2$ because 2 is the remainder after dividing 7 by 5 . For the same reason, $28 \bmod 5=3$. A hash data structure based on "mod $5 "$ will map 28 to bucket (or pool) 3, 7 to pool 2, 12 to pool 2, 59 to pool 4, and so on. There are many variants of hashing, some of which entail hashing each pool resulting from the first hash function in order to get "purer" pools, and then using the combined hash results to generate an item "label". For example, if there were a second hash function, this time based on "mod 7 ", then 28 would map to 0 and 53 to 5. Thus the full "label" of 28 would be $(3,0)$ because $28 \bmod 5=3$ and 28 $\bmod 7=0$. By contrast, the label of 53 would be $(3,4)$ because $53 \bmod 5=3$ and $53 \bmod 7$ is 4 . Associated with each unique label is a different pool usually having a small number of distinct values.

DNA hash pooling or hash pooling for short is the analogous operation on DNA. The "hash functions" in this scenario correspond to biological operations that give rise to distinctive and quantifiable "fingerprints" (e.g., measurement of GC content followed by digestion by a set of restriction enzymes). The label components correspond to the "values" obtained by application of the hash functions (e.g. GC content and fragment length). 


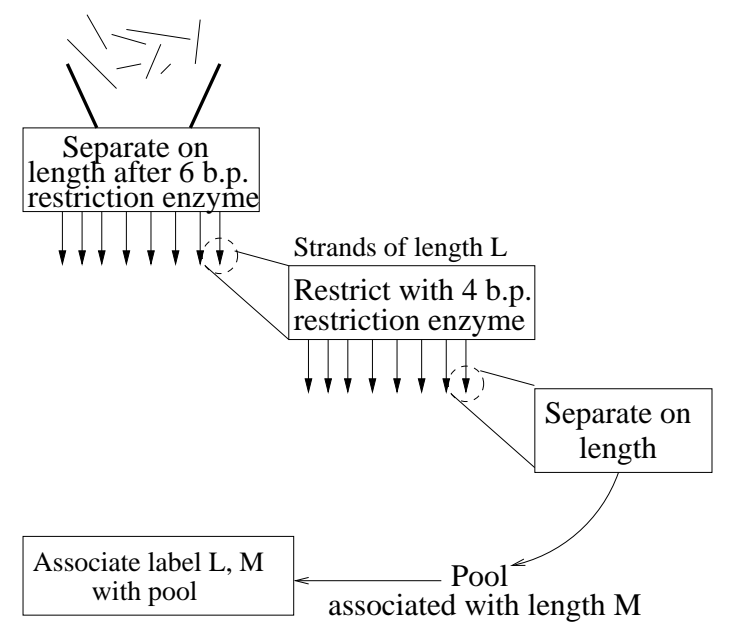

Figure 1: Two-stage hash pooling

In silico, our method involves simulating these operations on known sequences (typically of entire genomes) and characterizing different portions of those sequences from the result(s). In vitro, our method involves performing the analogous, bench-based operations and sequencing only those pools that are likely to be pure (this is, unique to one genome), or otherwise of interest. Such pools tend to pertain to long lengths, as we shall see later.

For concreteness, this paper focusses on hash pooling based solely on restriction enzymes. The basic operations are

1. Apply a six base-pair (b.p.) restriction enzyme to a sequence, yielding a set of fragments.

2. Partition those fragments based on length (perhaps approximately), using a technique such as gel electrophoresis.

3. Apply a four b.p. restriction enzyme to each partition (or a selected subset of partitions) and separate on length again.

Each resulting pool is therefore associated with a label consisting of two lengths, the first based on a six b.p. restriction enzyme and the second based on a four b.p. restriction enzyme (Figure 11). 
The algorithm may be described in pseudo-code as follows:

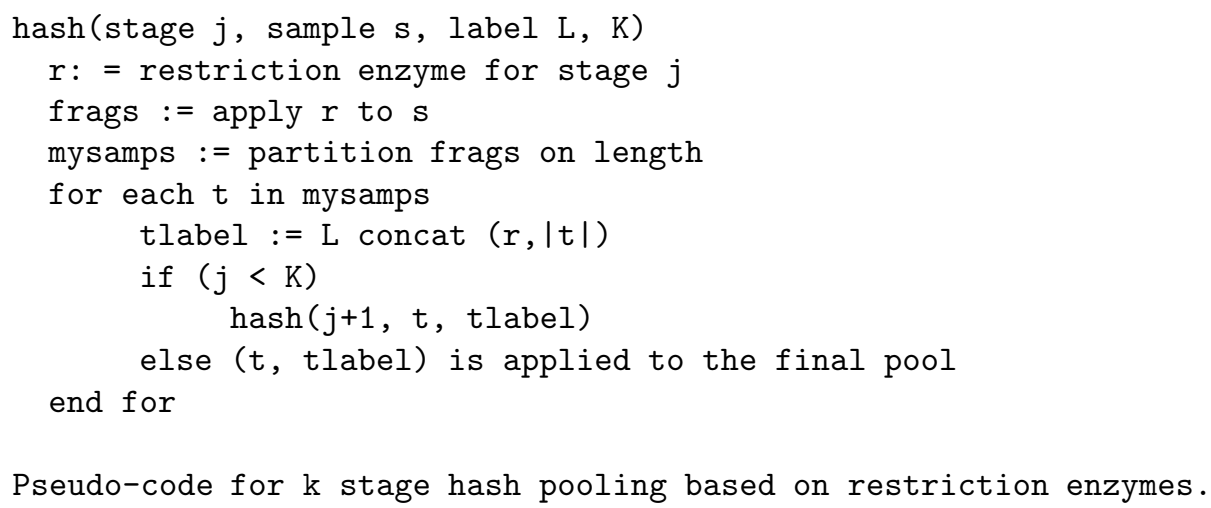

Pseudo-code for $\mathrm{k}$ stage hash pooling based on restriction enzymes.

For example consider the genomic sequence of bacterium A (E. coli K12). If we cut A using the enzyme SmaI (recognising CCCGGG), take the pool corresponding to length 264, cut that pool with RsaI (GTAC) and take the pool of length 31 , we get a pool having label $(264,31)$, its only member being the sequence CTATCCGCTCAATGAGTCGGTCGCCATTGCC. By contrast, the pool with label $(770,207)$ has three different sequences. For some applications, we will want pools having singletons (i.e., a set with only a single element) in order to obtain a pure sequence without the need for cloning.

One may object that separating fragments by length entails a certain inaccuracy imposed by the laboratory technique; a reasonable estimate of this error may be plus or minus 10 b.p. In this case, in order to obtain a pure sample, we may be interested in finding a pool whose label has no "10 b.p.-neighbors." The labels $L$ and $L^{\prime}$ are 10 b.p.-neighbors if (i) the first component of $L$ and the first component of $L^{\prime}$ are different but differ by 10 or less $\left(0<\left|L[0]-L^{\prime}[0]\right| \leq 10\right)$; or (ii) the first component of $L$ and $L^{\prime}$ are the same but the second components differ by 10 or less $\left(\left|L[1]-L^{\prime}[1]\right| \leq 10\right)$. For $E$. coli K12, the labels $(188,59)$ and $(188,106)$, for example, have no 10 b.p.-neighbors.

\section{Experiments}

Having presented our formal framework, we can now present several applications and our in silico results.

\subsection{Large Genome Sequence Detection}

The question we ask is the following: given a tube, $T$, of unknown DNA (perhaps from an environmental sample) and a genome whose sequence is known, are "reasonably sized" portions of that genome present in $T$, even if in small concentrations? (Figure 22)

A "reasonably sized" portion is a sequence of length at least 200,000 b.p. (which might account for roughly $5 \%$ of the size of a bacterial genome.) This might be used for the detection of bacterial pathogens in food, for example. In what follows, we used bacterium A, E. coli K12, which is often used as an indicator organism in the detection of faecal contamination. Our in silico experiment involved the following steps: 


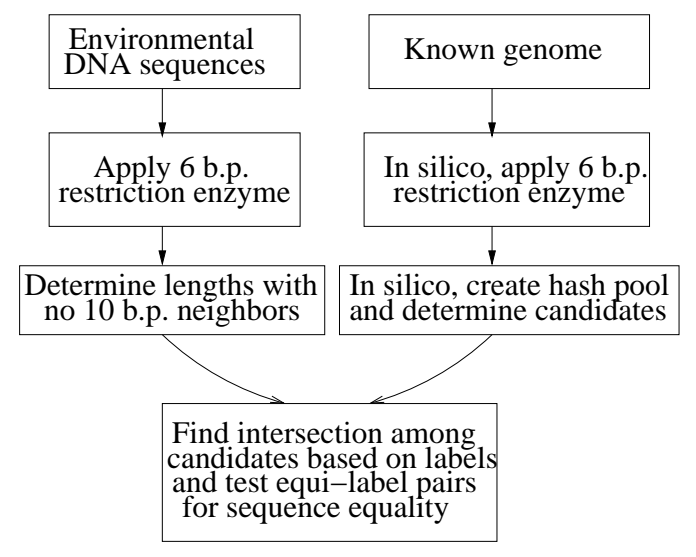

Figure 2: Genome sequence detection

1. Compute the candidate set of A, based on no 10 b.p. neighbors. There were 3,567 candidates (final pools from A having no 10 b.p. neighbors). This gives us a "comparison library" of pools. It is important to note that this is a "once only" operation for any combination of known genome and restriction enzyme set.

2. Take a 200,000 consecutive b.p. subsequence of A. (with the start position taken uniformly at random) and combine it with a sequence of length four times that of A (generated pseudo-randomly to have the same GC content as A.) Compute its candidate set of hash pools, based on no 10 b.p. neighbors.

Having tried this 20 times, we found, on average, 2,000 pools in the second candidate set. On average the two sets had an intersection of cardinality 71 based on their labels. When labels were equal, $99.8 \%$ of the time there was a match of the sequence and the sequence was in bacterium $\mathrm{A}$.

When applying this in a laboratory setting, there is the significant question of whether this operation requires many rounds of DNA digestions. Fortunately, the answer is no. For each of the 20 tests, the six b.p. restriction enzyme was used and, on average, 5.8 fragment lengths were chosen, 4.7 of which (on average) yielded matching sequences. So, if this were done in vitro, approximately 70 common strands would be found using one application of SmaI and approximately six applications of RsaI. Virtually all tested strands would be shown to be equal (so sequencing would not even be necessary).

Martyn: we should test this for sure

When the neighbor condition is reduced to 5 b.p., E. coli yields 6,008 candidate pools. The comparison group (again 200,000 bases from A and a pseudorandom sequence four times the length of $\mathrm{A}$ based on the same GC content) yielded approximately 6,500 candidate pools each time, based on no 5 b.p. neighbors. On average the two sets had an intersection of cardinality 150 based on their labels. When labels were equal, $99.2 \%$ of the time there was a match of the sequence and the sequence was in bacterium A. In each of the 20 tests, the six b.p. restriction enzyme was used and then, on average, 8.75 fragment lengths were chosen, 6.75 of which (on average) yielded matching sequences. 


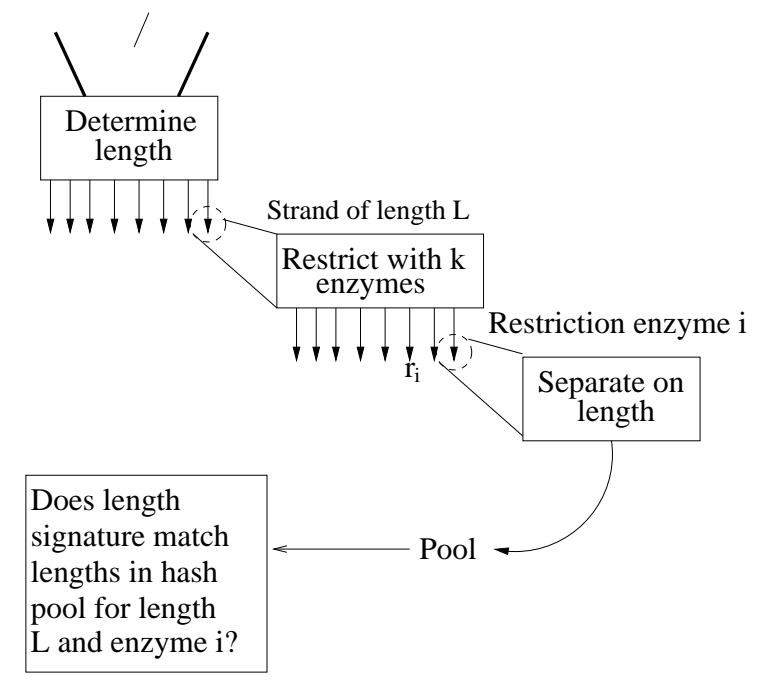

Figure 3: Sequence querying

This experiment shows that in silico hash pooling on a known genome can identify pools to look for in a sample, such that those pools have a strong likelihood of containing a subsequence of the known genome. Thus, we can see this method as an improvement over random sampling, and can be used even if the bacterium of interest is relatively rare in the sample.

\subsection{Sequence Detection}

Here we address the question: given a query sequence, is that sequence present, at least in part, in the tube? This might be used to look for the presence of a pathogen, for example. This question is clearly related to the previous one. In fact, an experiment similar to that used to address the first question serves as an illustration: suppose the sample under scrutiny contains A plus a lot of other assorted DNA (the full genome of A amongst a pseudo-random sequence four times the length of the A sequence.) Then, 20 times, we take a random query subsequence of length 200,000 from A and see if we can find matching parts in the sample tube.

The sample tube (A sequence plus a random sequence four times $\mathrm{A}$ in length with no 10 b.p. neighbors) has 3,567 candidate pools. The average 200,000 b.p. subsequence of $\mathrm{A}$ has about 200 candidates. If two labels are equal, the corresponding sequences matched $100 \%$ over all 20 tests of length 200,000 .This is not guaranteed, but again shows that relatively little sequence is likely to be required to find a matching subsequence of the query string if it is present. If the query string is not present in the sample at all, then very few corresponding pools will be found.

\subsection{Sample Comparison}

Here we consider the problem: given two tubes of DNA, do they contain strands that are the same or very similar? This might be useful when comparing samples 


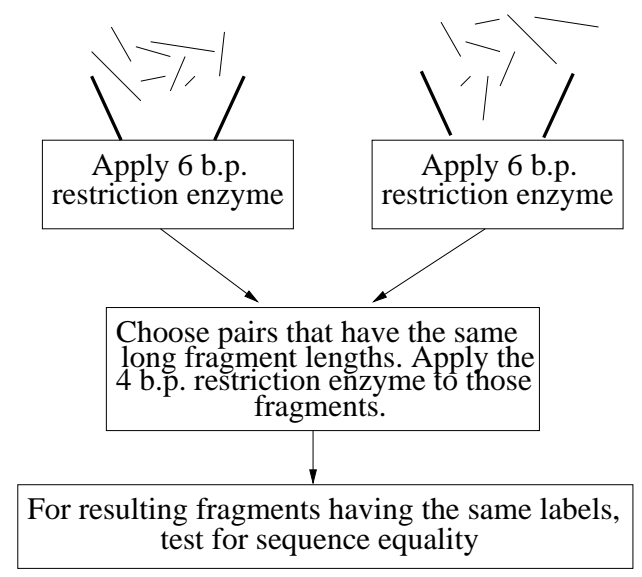

Figure 4: Sample comparison

of unsequenced genomes. In this case, we cannot compute candidate pools that have no b.p. neighbors, because we do not have access to full genomic sequences. However, we can choose the most likely pairs to study by focussing on "unusual" labels (Figure 4). We therefore performed the following in silico experiment:

1. Take a 200,000 b.p. sequence, target, with the same GC content as A, plus a random sequence four times the size of $\mathrm{A}(4 \times 4.7 M b=\approx 20 M b)$, with the same average GC content as A.

2. For the second sample, we use target plus another random sequence four times the size of $\mathrm{A}$, with the same average GC content. Thus the target in each sample is 200,000 b.p. long, compared to roughly 20 million for the entire sequence present in each tube.

Now the question is this: in which pools should we look for common strands? That is, is it better to look at pools where the six b.p. restriction enzyme has cut strands of length approximately 4,000 (the expected value) or much longer? The in silico answer is obvious in retrospect: to find common strands, the best pools to look at are ones corresponding to long lengths when cut by the first restriction enzyme. Thus the procedure is this:

1. Cut each sample with the six b.p. restriction enzyme, then find all lengths that are the same (within an accuracy of 10 b.p.).

2. On the upper quartile of those lengths (approximately 235 of them), apply the four b.p. restriction enzyme.

Of those fragments that have the same lengths (within 10 b.p.) for both the first and second restriction enzymes, between $4 \%$ and $7 \%$ are the same sequence over the 20 experiments that we tried. Other quartiles are about a factor of 10 less good. On the other hand, if we look at dectiles $(1 / 10)$, then the upper $1 / 10$ of the lengths (from 10,210 to 24,550 ) gives a hit ratio of about $14 \%$, and only 94 lengths from the six b.p. restriction enzyme require an application of the second restriction enzyme. 


\subsection{Vector Candidate Sequence Identification}

Given a tube of strands from one or more known genomes, can we find a pure strand of several thousand? This might be useful when trying to assemble vectors for DNA insertion, for example. Given what we already know, this is very easy: we simply find sequences with no 10 b.p. neighbors.

\section{$5 \quad$ Implementation Issues}

In this section we give a brief overview of the two main laboratory tools from which our basic operations are built: restriction enzymes (for chopping DNA into sections) and gel electrophoresis (for sorting fragments according to length). The descriptions are taken from [?].

\section{$5.1 \quad$ Restriction Enzymes}

Restriction endonucleases [?] (often referred to as restriction enzymes) recognize a specific sequence of DNA known as a restriction site. Any DNA that contains the restriction site within its sequence is cut by the enzyme at that point 1

(a)

5' G-G-A-T-G-T-A-C-G-G-T-A 3'

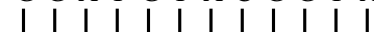

3' C-C-T-A-C-A-T-G-C-C-A-T 5'

5' G-G-A-T-G-T A-C-G-G-T-A 3'

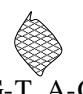

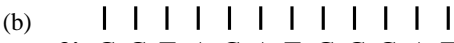

3' C-C-T-A-C-A T-G-C-C-A-T 5'

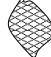

5' G-G-A-T-G-T A-C-G-G-T-A 3'

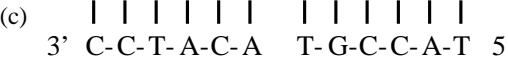

Figure 5: (a)Double-stranded DNA. (b) DNA being cut by RsaAI. (c) The resulting blunt ends

For example, the double-stranded DNA in Fig. 5a is cut by restriction enzyme RsaI, which recognizes the restriction site $G T A C$. The enzyme breaks (or "cleaves") the DNA in the middle of the restriction site (Fig. 5b). The exact nature of the break produced by a restriction enzyme is of great importance. Some enzymes like RsaI (mentioned earlier) leave "blunt" ended DNA (Fig. 5r). Others may leave "sticky" ends. For example, the double-stranded DNA in Fig. 6a is cut by restriction enzyme Sau3AI, which recognizes the restriction site GATC (Fig. 6b). The resulting sticky ends are so-called because they are then free to anneal to their complement. It is important to note that we use only blunt-ended restriction enzymes, in order to prevent erroneous annealing.

\footnotetext{
${ }^{1}$ In reality, only certain enzymes cut specifically at the restriction site, but we take this factor into account when selecting an enzyme.
} 


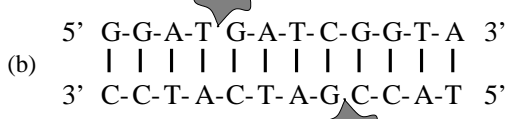

Figure 6: (a) Double-stranded DNA being cut by Sau3AI. (b) The resulting sticky ends

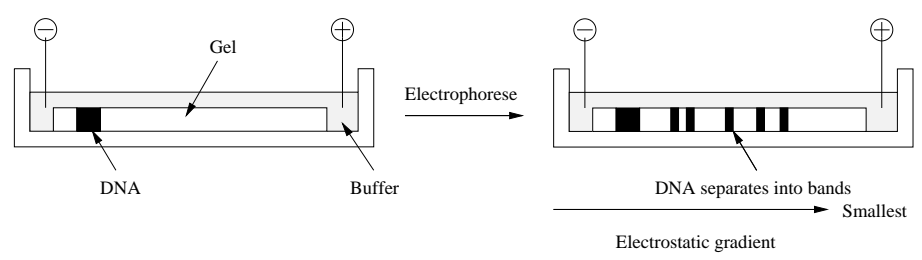

Figure 7: Gel electrophoresis process

\subsection{Gel Electrophoresis}

Gel electrophoresis is an important technique for sorting DNA strands by size [?]. Electrophoresis is the movement of charged molecules in an electric field. Since DNA molecules carry a negative charge, when placed in an electric field they tend to migrate toward the positive pole. The rate of migration of a molecule in an aqueous solution depends on its shape and electric charge. Since DNA molecules have the same charge per unit length, they all migrate at the same speed in an aqueous solution. However, if electrophoresis is carried out in a gel (usually made of agarose, polyacrylamide, or a combination of the two), the migration rate of a molecule is also affected by its size.2 This is due to the fact that the gel is a dense network of pores through which the molecules must travel. Smaller molecules therefore migrate faster through the gel, thus sorting them according to size.

A simplified representation of gel electrophoresis is depicted in Fig. 7. The DNA is placed in a well cut out of the gel, and a charge applied.

Once the gel has been run (usually overnight), it is necessary to visualize the results. This is achieved by staining the DNA with the fluorescent dye ethidium bromide and then viewing the gel under ultraviolet light. At this stage the gel is usually photographed.

One such photograph is depicted in Fig. 8. Gels are interpreted as follows; each lane (1-7 in our example) corresponds to one particular sample of DNA (we use the term tube in our abstract model). We can therefore run several tubes

\footnotetext{
$[?]$.

${ }^{2}$ Migration rate of a strand is inversely proportional to the logarithm of its molecular weight
} 


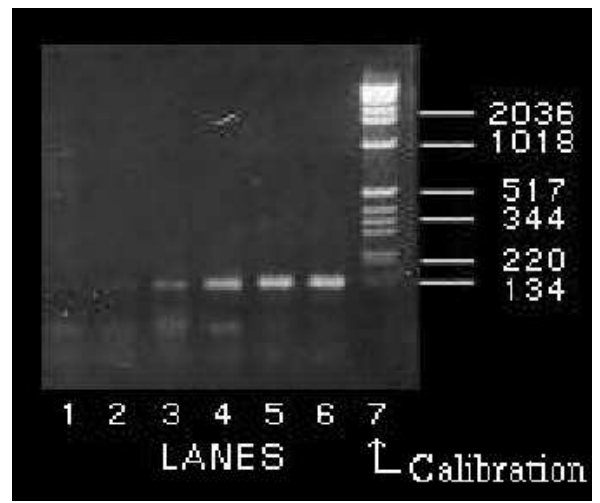

Figure 8: Gelelectrophoresis photograph

on the same gel for the purposes of comparison. Lane 7 is known as the marker lane; this contains various DNA fragments of known length, for the purpose of calibration. DNA fragments of the same length cluster to form visible horizontal bands, the longest fragments forming bands at the top of the picture, and the shortest ones at the bottom. The brightness of a particular band depends on the amount of DNA of the corresponding length present in the sample. Larger concentrations of DNA absorb more dye, and therefore appear brighter. One advantage of this technique is its sensitivity - as little as $0.05 \mu \mathrm{g}$ of DNA in one band can be detected as visible fluorescence.

The size of fragments at various bands is shown to the right of the marker lane, and is measured in base pairs (b.p.). In our example, the largest band resolvable by the gel is 2,036 b.p. long, and the shortest one is 134 b.p. long. Moving right to left (tracks $6-1$ ) is a series of PCR reactions which were set up with progressively diluted target DNA (134 b.p.) to establish the sensitivity of a reaction. The dilution of each tube is evident from the fading of the bands, which eventually disappear in lane 1.

\section{Conclusions}

